\title{
Morphological, Electrical and Mechanical Properties of Ultrahigh Molecular Weight Polyethylene and Multi-wall Carbon Nanotube Composites Prepared in Decalin and Paraffin
}

\author{
Yuezhen BIN $^{\dagger}$ Atsuko YAMANAKA, Qingyun CHEN, Ying XI, \\ Xiaowen JIANG, and Masaru MATSUO ${ }^{\dagger}$ \\ Department of Textile and Apparel Science, Faculty of Human Life and Environment, \\ Nara Women's University, Kitauoyanishi-machi, Nara 630-8263, Japan
}

(Received February 5, 2007; Accepted March 18, 2007; Published May 9, 2007)

\begin{abstract}
Ultrahigh molecular weight polyethylene (UHMWPE) and multi-wall carbon nanotube (MWNT) composites were prepared using either decalin or paraffin as solvent. Electrical conductivity measurements were performed for the original and heat-treated composites. The drastic increase in conductivity occurred at low MWNT content for the composite prepared in paraffin, while the conductivity of the composite prepared in decalin increased slightly up to $10 \mathrm{wt} \%$ MWNT content. Scanning electron microscopy observations revealed that the MWNTs within the composite prepared in decalin were covered by UHMWPE, and their average diameters were much greater than those of the original MWNTs, while the average diameter of the MWNTs within the composite prepared in paraffin was similar to the diameter of the original MWNTs. Such different morphology was found to be due to the different crystallization. The composites prepared in decalin had high drawability. Interestingly, the conductivities of the drawn (up to 50 times) and undrawn UHMWPE-MWNT composites were almost equal, independent of the MWNT content. As for the UHMWPE-MWNT composite (drawn up to 50 times) with $15 \mathrm{wt} \%$ MWNT content, the conductivity reached up to $10^{-1} \mathrm{~S} / \mathrm{cm}$, and the modulus was higher than $50 \mathrm{GPa}$ at $c a .25^{\circ} \mathrm{C}$, indicating that a polymeric material with high modulus and relative high conductivity was successfully prepared. [doi:10.1295/polymj.PJ2006229]

KEY WORDS Ultrahigh Molecular Weight Polyethylene / Multi-Wall Carbon Nanotubes /

Decalin / Paraffin / Electrical Conductivity /
\end{abstract}

Carbon nanotubes (CNTs) have attracted great interest since their discovery. CNTs are long and slender fibers with hexagonal carbon (graphite structure) walls, which are often capped at the two ends. CNTs possess superior mechanical, thermal and electrical properties, and so offer tremendous opportunities for developing new fundamental material systems. ${ }^{1-3}$

The processing of $\mathrm{CNT} /$ polymer composites has been reported in a number of papers, but this field is still in its infancy. Significant issues regarding the purification and dispersion of CNTs, and the bulk processing of these materials still remain. The ability to disperse CNTs into a polymer may be the most critical processing parameter for controlling the properties of the composites. ${ }^{4-11}$ Fox example, Zhang et al. reported a new method for dispersing SWNT bundles in UHMWPE by spraying an aqueous solution of these single-wall carbon nanotubes containing sodium dodecylbenzene sulfate as surfactant onto UHMWPE powder, and then a composite film was prepared from the UHMWPE powder dissolved in xylene. ${ }^{9}$ The most popular method for preparing CNT/polymer composites involves the direct mixing of the CNTs into the polymer with a twin-screw melt mixer. ${ }^{10,11}$ However, this method is limited for highly viscous polymer materials.

Polymer/multi-wall carbon nanotubes (MWNT) interactions include (a) multicontacts between the polymer chains on the surface of the MWNTs (physicosorption), (b) reactions of the MWNTs with polymers (chemisorption), and (c) mechanical chain entanglements and covalent carbon-carbon cross-links within the polymer matrix. ${ }^{12,13}$ These interactions obviously influence the electrical conductivity and mechanical properties of the composites. The uniform mixing of MWNTs into UHMWPE by gelation/crystallization from solution depends on the structure of the curved graphene sheets of the MWNTs. MWNTs with few defects in the curved graphene sheets induced hard dispersion into the UHMWPE matrix, although this problem was overcome by the admixture of the ethylene-methylmethacrylate (EMMA) copolymer. ${ }^{14}$ On the other hand, the uniform mixing of MWNTs (having a number of defects in the curved graphene sheets) into UHMWPE has been achieved by the described gelation/crystallization method. ${ }^{15}$

This paper deals with the characteristics of UHMWPE-MWNT composites prepared by using

\footnotetext{
${ }^{\dagger}$ To whom correspondence should be addressed (Tel/Fax: +81-742-20-3462, E-mail: yuezhen@cc.nara-wu.ac.jp (Y. Bin) m-matsuo@cc.nara-wu.ac.jp (M. Matsuo)).
} 
MWNTs with many defects in curved graphene sheets. In this experiment, to ensure uniform mixing of MWNTs into UHMWPE, the sample preparation was performed using either decalin or paraffin as solvent. Sample preparation by decalin was adopted as an example of gelation/crystallization from solution. On the other hand, the sample preparation by paraffin was adopted instead of films molded at the melt state, since the film preparation by melting of UHMWPE powder at high temperature was impossible because of extremely high viscosity of the melt state. The characteristics of the composites prepared by the two different methods were investigated in terms of the morphological aspects of the composites, although analysis as a function of draw ratio was limited for the composite prepared in decalin.

\section{EXPERIMENTAL}

UHMWPE (Mitsui Chemicals, Hizex) with an average viscosity molecular weight $\left(M_{\mathrm{v}}=6,300,000\right)$, and fibrous-type MWNTs, were used as the test specimen. MWNTs used in our experiment are Hyperion Graphite Fibrils with diameter (D) of 10-20 nm, and an aspect ratio (L/D) of $1-2 \times 10^{3}$. The BET surface area of the MWNTs is $250 \mathrm{~m}^{2} / \mathrm{g}$, and the DBP adsorption is $400-500 \mathrm{~cm}^{3} / 100 \mathrm{~g}$. The true density is $2.0 \mathrm{~g} / \mathrm{cm}^{3}$.

Figure 1 shows a scanning electron microscopy (SEM) image and Raman spectrum (excitation laser wavelength of $488 \mathrm{~nm}$ ) of the original MWNTs. Figure 1a indicates the MWNTs possess high purity and a uniform diameter distribution. In the Raman spectrum of Figure $1 \mathrm{~b}$, however, the peak of the Raman-allowed phonon mode, $E_{2 \mathrm{~g}}$, at about 1582 $\mathrm{cm}^{-1}$ is not very sharp, while the intensity of the peak at $1345 \mathrm{~cm}^{-1}$ is high, and this peak appears through the disorder-induced phonon mode due to the infinite size of the crystals and defects. This result indicates the present MWNTs have a low degree of graphitization.

Two different solvents were used in the preparation of the UHMWPE-MWNT composites. One is decalin and the other, paraffin $\left(\mathrm{C}_{16}-\mathrm{C}_{21}\right)$. The methods employed in preparing the samples using the two kinds of solvent were quite different.

When decalin was used as the solvent, the MWNTs were firstly ultrasonicated in the decalin for more than $5 \mathrm{~h}$ at room temperature. Then, UHMWPE was added and the mixture stirred and heated with a mild heating rate up to $140^{\circ} \mathrm{C}$, and maintained at this temperature for $1 \mathrm{~h}$. The concentration of UHMWPE was fixed at $1 \mathrm{~g} / 100 \mathrm{~mL}$ of decalin, which was higher than the optimum concentration of UHMWPE, $0.4 \mathrm{~g} / 100 \mathrm{~mL}$ of decalin, for the maximum drawability. ${ }^{16}$ However, the high concentration resulted in a highly viscous
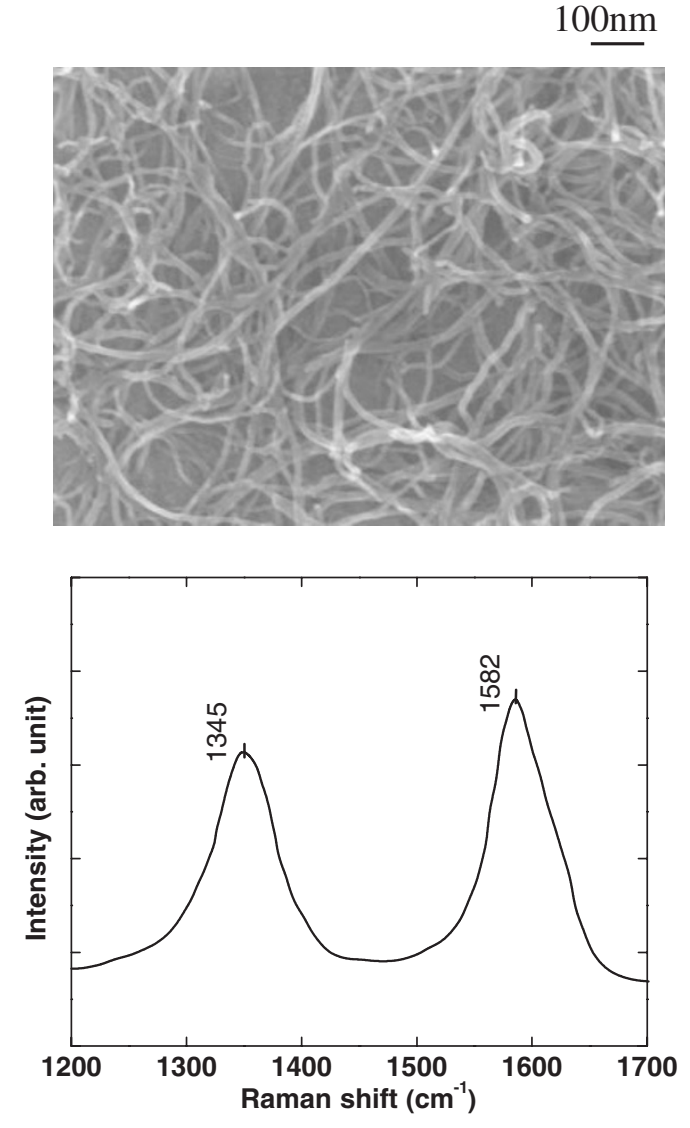

Figure 1. (a) SEM image and (b) Raman spectrum of original MWNTs.

solution, which provided strong shear forces to make MWNTs well-dispersed in solution. The volume of solution was controlled to obtain a film with thickness of $700-800 \mu \mathrm{m}$. The hot homogenized solution was quenched by pouring it into a glass dish at room temperature, thus generating a gel. The decalin solvent was evaporated from the gel under ambient conditions or at $60^{\circ} \mathrm{C}$. The latter evaporation was done to shorten the preparation time of the composites. The gel was further dried on an oven plate at $60^{\circ} \mathrm{C}$ when about $60 \%$ of the solvent had been evaporated at room temperature, forming a relative tough gel. Incidentally, such gelation/crystallization of UHMWPE (no MWNTs) using decalin as a solvent has been reported by $\mathrm{Smith}^{17,18}$ et al., while a single-crystal method similar to the gelation/crystallization method described has been reported by Kanamoto et al. ${ }^{19}$ The evaporation of solvent at $60^{\circ} \mathrm{C}$ from the composite is different from the previous result. ${ }^{16}$ However, the evaporation was demonstrated to achieve a good uniform UHMWPE gel. ${ }^{20}$ The composite film was cut into strips of length $30 \mathrm{~mm}$ and width $10 \mathrm{~mm}$. The strips were clamped in a manual stretching device and stretched in a hot oven at $135^{\circ} \mathrm{C}$.

When paraffin was used as solvent, the MWNTs were first stirred with UHMWPE in paraffin at room 
temperature for $48 \mathrm{~h}$ affording a black glossy paste. Then, the mixture was continually stirred and heated up to $250{ }^{\circ} \mathrm{C}$ at a mild heating rate of about $10^{\circ} \mathrm{C} /$ min, and maintained for $30 \mathrm{~min}$ under nitrogen gas. The concentration of UHMWPE prepared in this manner was determined to be $5 \mathrm{~g}$ against $20 \mathrm{~g}$ paraffin. This was the optimum concentration of UHMWPE to make MWNTs well-dispersed in solution and to form uniform films. The hot homogenized mixture was similar to the melted state of polymer rather than the solution. The mixture was then moved to a glass beaker containing benzene solvent at room temperature. The benzene was used to remove paraffin from the composites. After removal of the paraffin, the mixture was pressed between flat aluminum plates with mirror-finished surfaces under a pressure of $2 \mathrm{MPa}$ at $180{ }^{\circ} \mathrm{C}$ for $20 \mathrm{~min}$, and then cooled slowly to room temperature. The prepared sheets were about $1.2 \mathrm{~mm}$ thick.

The electrical conductivity at both room temperature and in the temperature range from $40^{\circ} \mathrm{C}$ to $160^{\circ} \mathrm{C}$ at a $5^{\circ} \mathrm{C} / \mathrm{min}$ heating rate, were measured using a high resistance measuring device (HR-100, Iwamoto Seisakusho Co. Ltd.). A digital multimeter was used (Advantest R6441A Digital Multimeter) when the resistivity was lower than $10^{7} \Omega \mathrm{cm}$. On the other hand, the measurements were done by using a high resistance meter (HP 4339B High Resistivity Meter) when the resistivity exceeded beyond $10^{7}$ $\Omega \mathrm{cm}$. The results were presented as a logarithm of electrical conductivity $(\log (\mathrm{S} / \mathrm{cm}))$.

$\mathrm{X}$-Ray measurements were carried out with a 12 $\mathrm{kW}$ rotating-anode X-ray generator (Rigaku RDArA) operated at $150 \mathrm{~mA}$ and $40 \mathrm{kV}$ with monochromatic $\mathrm{CuK} \alpha$ radiation (wavelength of $0.154 \mathrm{~nm}$ ). The intensity distribution was measured with a stepscanning device at step intervals of $0.05^{\circ}$, each at a fixed time of $100 \mathrm{~s}$, in the given range of twice the Bragg angle. Wide angle X-ray diffraction (WAXD) patterns were obtained using a flat camera, while small angle X-ray scattering (SAXS) measurements were obtained using a position-sensitive proportional counter (PSPC). The temperature dependence of the dynamic tensile modulus was estimated with a viscoelastic spectrometer (VES-F) (Iwamoto Machine Co., Ltd.) at a fixed frequency of $10 \mathrm{~Hz}$ over the temperature range of $-150-300{ }^{\circ} \mathrm{C}$. The fibril structure was observed by scanning electron microscopy (SEM, TOPCON, ABT150F).

\section{RESULTS AND DISCUSSION}

To investigate the influence of the MWNT content on the orientation of the UHMWPE crystallites and lamellae, WAXD and SAXS measurements were car-

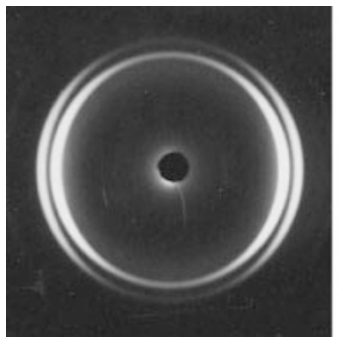

(a) MWNTs= 0 wt $\%$

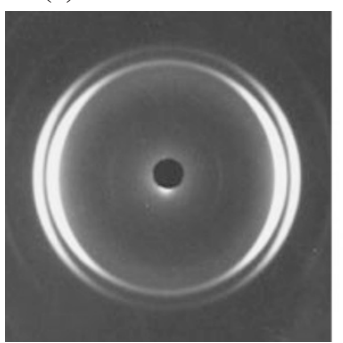

(b) MWNTs $=0.25 \mathrm{wt} \%$

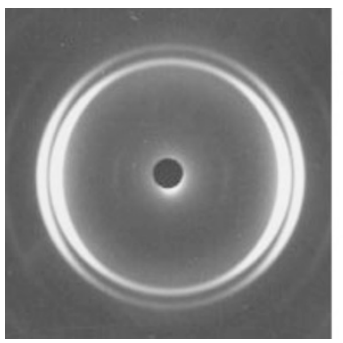

(c) $\mathrm{MWNTs}=2 \mathrm{wt} \%$

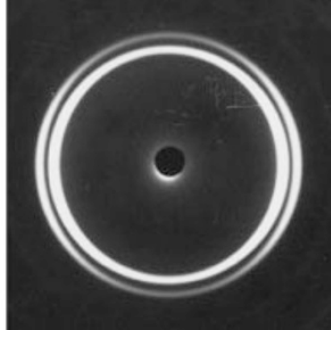

(d) MWNTs $=3 \mathrm{wt} \%$

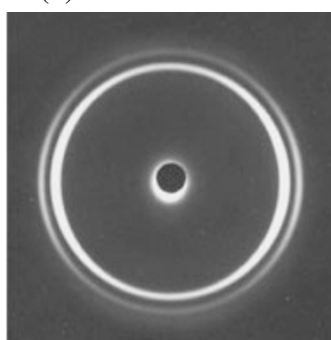

(e) MWNTs $=8 w t \%$

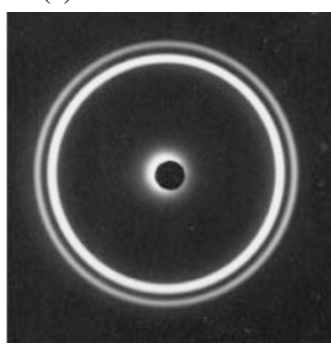

(f) $\mathrm{MWNTs}=15 \mathrm{wt} \%$
Figure 2. WAXD patterns (edge view) for the undrawn composites.

ried out for UHMWPE-MWNT composite films containing different contents of MWNTs. Figure 2 shows WAXD patterns (end view) for the undrawn composite films prepared using decalin as a solvent, when an incident beam was directed parallel to the film surface. The diffraction arcs from the (110) and (200) planes of the UHMWPE crystallites indicate that the $c$-axes are oriented perpendicular to the film surface, but the orientation degree becomes blur by the introduction of MWNTs, especially for the composites containing more than $3 \mathrm{wt} \%$ MWNT. The diffraction from the composite containing $15 \mathrm{wt} \%$ MWNTs indicates a random orientation of UHMWPE crystallites. In addition, any diffraction arcs (and rings) from the (002) planes of the MWNTs were not observed for any of the composite films with MWNT content, because of the very strong diffraction intensity from the (110) and (200) planes of the UHMWPE. Incidentally, the WAXD patterns for the UHMWPE film $(0 \%)$ prepared in paraffin showed diffraction rings indicating no preferential orientation of the $c$-axes of the UHMWPE crystallites, and the WAXD patterns for the composites containing 15\% MWNTs also showed the same diffraction rings as shown in pattern (f).

Figure 3 shows the corresponding SAXS intensity 


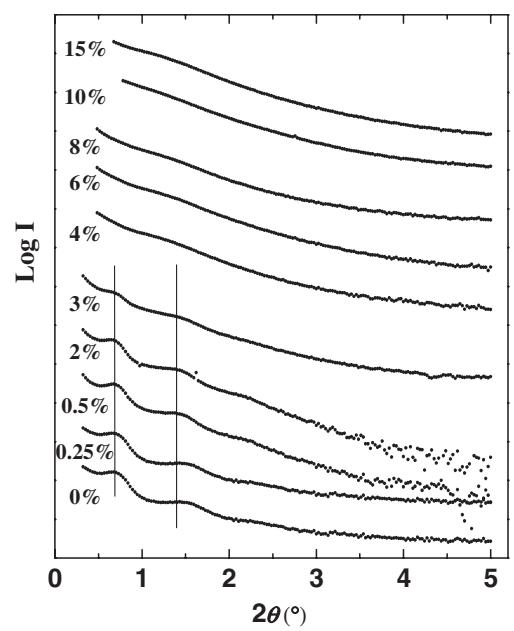

Figure 3. SAXS intensity distribution for the undrawn composite film in the meridian direction by PSPC system.

distribution (prepared in decalin) measured for the undrawn composite films in the meridian direction using the PSPC system. The profiles of the composite films with an MWNT content less than $3 \mathrm{wt} \%$ show that the profile of the intensity distribution was similar to that of the UHMWPE homopolymer film (MWNTs = $0 \%$ ). The two or three orders of scattering maxima were confirmed from the profiles, indicating that the dry composite films were composed of highly-oriented crystal lamellae with their flat faces parallel to the film surface. When the MWNT content was beyond the percolation threshold of $3 \mathrm{wt} \%$, the scattering maxima began to disappear, indicating that the lamellae were oriented randomly for the composites with 10 and $15 \mathrm{wt} \%$ MWNTs. As for the UHMWPE composites, with a MWNT content of less than $3 \mathrm{wt} \%$, the results of the WAXD patterns in Figure 2 together with the SAXS intensity distributions in Figure 3, indicated that the dry gel was composed of crystalline lamellae oriented predominantly with their large flat faces parallel to the film surface in a manner similar to that observed for mats of single crystals. Such orientation behavior of the lamellae became less pronounced with increasing MWNT content. When MWNTs were admixed beyond $10 \mathrm{wt} \%$, the scattering maximum disappeared and the distribution showed a monotonical curve characterizing the random orientation of the lamellae. Incidentally, no peak was also observed for the composites prepared in paraffin, indicating a random orientation of the crystal lamellae. Because, the molded films were prepared by compression under $2 \mathrm{MPa}$ at $180^{\circ} \mathrm{C}$ as described in Experimental section. Under such a compression, the preferential orientation of the crystal lamellae in the dry gel film prepared by decalin disappeared.

Figure 4 shows the electrical conductivity against MWNT content for the composites prepared in either

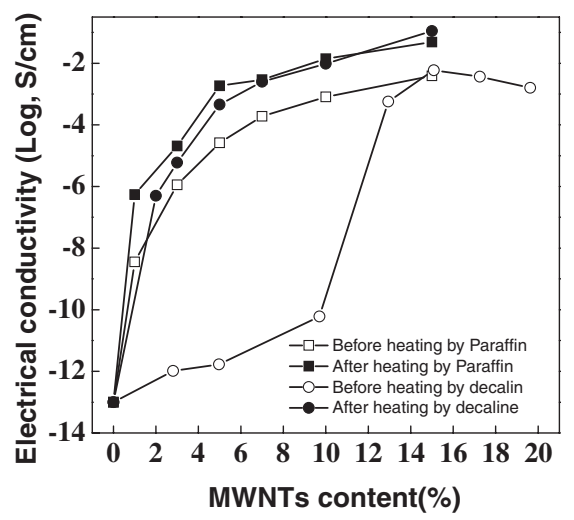

Figure 4. Electrical conductivity against MWNT content for the composite prepared from paraffin and decalin solvents.

paraffin or decalin solvent. The measurements were performed for both the original and heat-treated specimens. The drastic increase occurred at low MWNT content for the composite prepared using paraffin, and the electrical conductivity for each content was slightly higher after heat-treatment at $155^{\circ} \mathrm{C}$. On the other hand, the conductivity of the composite prepared using decalin solvent increases slightly up to $10 \%$ MWNT content, and increased drastically beyond $10 \%$. The conductivity also increased drastically as a result of the heat-treatment at $155^{\circ} \mathrm{C}$, where the obtained values are close to those of the composite prepared in paraffin solvent. In determining whether the percolation threshold of the original composite prepared in paraffin is much lower than that prepared in decalin, the thermal behavior of these composites was monitored by SEM observation.

Figure 5 shows cross-sectional areas of the UHMWPE-MWNT composite with a $3 \mathrm{wt} \%$ MWNT content prepared in decalin solvent, in which (a) and (b) are images of the composites before and after heat-treatment at $c a .150^{\circ} \mathrm{C}$. Interestingly, the MWNTs are covered by UHMWPE and their average diameters were much thicker than the original diameters of the MWNTs, indicating that most of the UHMWPE chains were crystallized on the MWNT surface acted as nuclear growth of UHMWPE. In contrast, the image (b) reveals that the average diameter of the MWNTs within the composite after heat-treatment showed a similar size to that of the original MWNTs shown in Figure 1a. Namely, the overlapped bare MWNTs are dispersed within the UHMWPE matrix. This means that most of the melted UHMWPE was removed from the MWNT surfaces, and recrystallization of UHMWPE under the cooling process did not occur on the MWNT surface. Accordingly, this phenomenon provided a drastic increase in the electrical conductivity of the composite after the heat-treatment, as shown in Figure 4. 


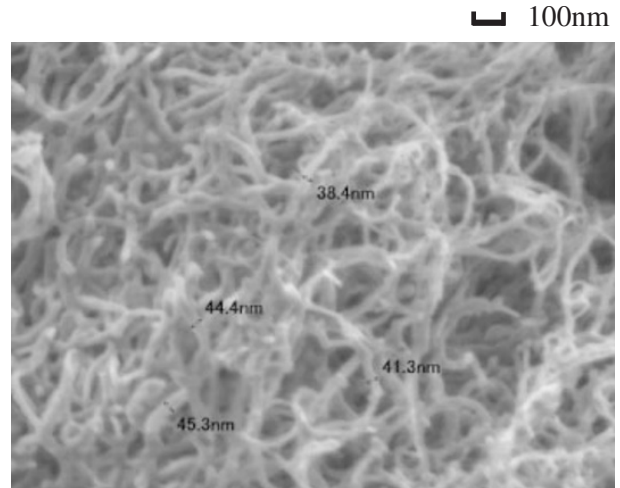

(a) decalin (before heating)

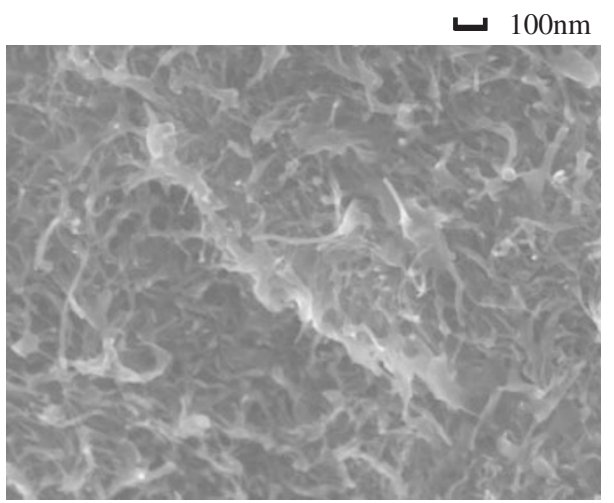

(c) paraffin (before heating)

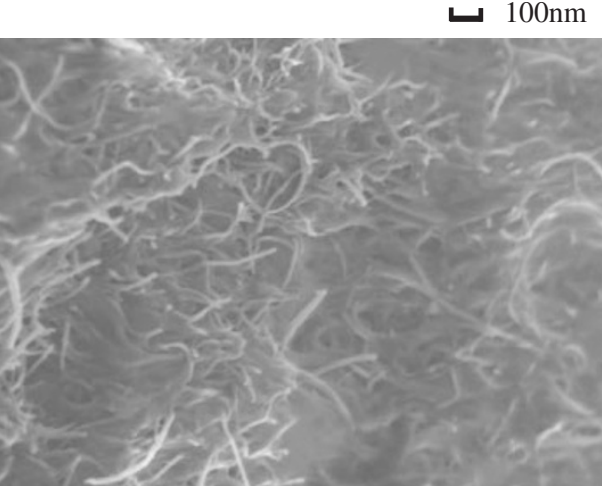

(b) decalin (after heating)

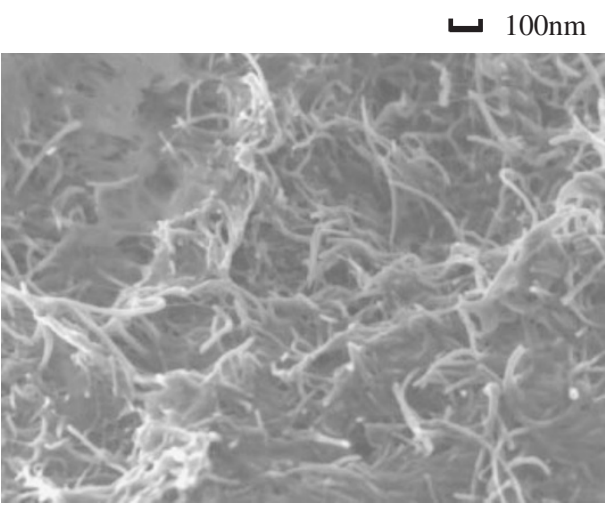

(d) paraffin (after heating)

Figure 5. SEM images observed on the cross section area of (a) original MWNTs and UHMWPE-MWNT composites with 3 wt $\%$ MWNT content.

On the other hand, images (c) and (d) show the cross-sections of the composite prepared in paraffin solvent, both before and after heat-treatments. It is seen that the average diameters of the MWNTs in the matrix are independent of the heat-treatment, and almost the same as the original MWNTs, indicating that most of the UHMWPE chains were not crystallized on the surface of the MWNTs. This is due to the fact that the sample preparation by paraffin is the same as the film preparation from melting state by heating like image (b). Hence, for the original composites, electron transfer between the adjacent MWNTs within the composite prepared in paraffine, became more active in comparison with the electron transfer between adjacent MWNTs in the matrix prepared in decalin. Then, the conductivity of the original composite prepared in decalin became lower than that for the matrix prepared in paraffin, as shown in Figure 4. As discussed before, the electrical conductivities of the two composites after heat-treatment were almost the same at the indicated MWNT contents, as shown in Figure 3, and that the MWNTs in both composites also showed similar average diameters. Namely, the diameter of the MWNTs in the composite prepared using decalin decreased drastically as a result of heat-treatment.

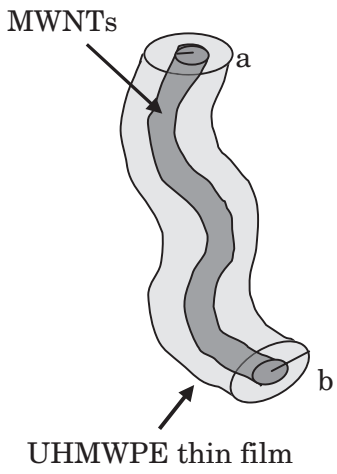

Figure 6. Schematic diagram of the composite prepared by decalin solvent proposed from the SEM images in Figure 5.

Figure 6 shows a schematic diagram of the composite prepared in decalin solvent proposed on the basis of the SEM images in Figure 5, in which $a$ and $b$ are the average radii of the original MWNTs and of the MWNTs covered by UHMWPE within the original composite (before heat-treatment). Based on the SEM images, $a$ and $b$ were determined to be 8.5 $(17 / 2)$ and $21(42 / 2) \mathrm{nm}$, respectively. Thus the volume fraction of covered UHWMPE becomes $83.6 \%$. On the other hand, the volume fraction of UHMWPE corresponds to $c a$. $96.7 \%$, calculated on the basis of 
(a) By decalin
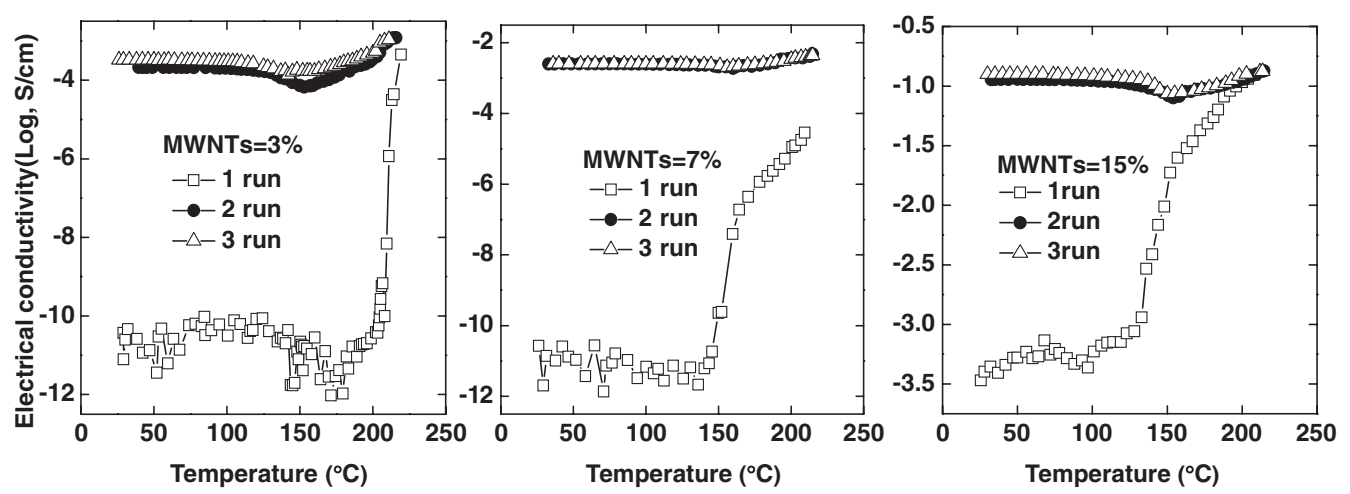

(b) By paraffin
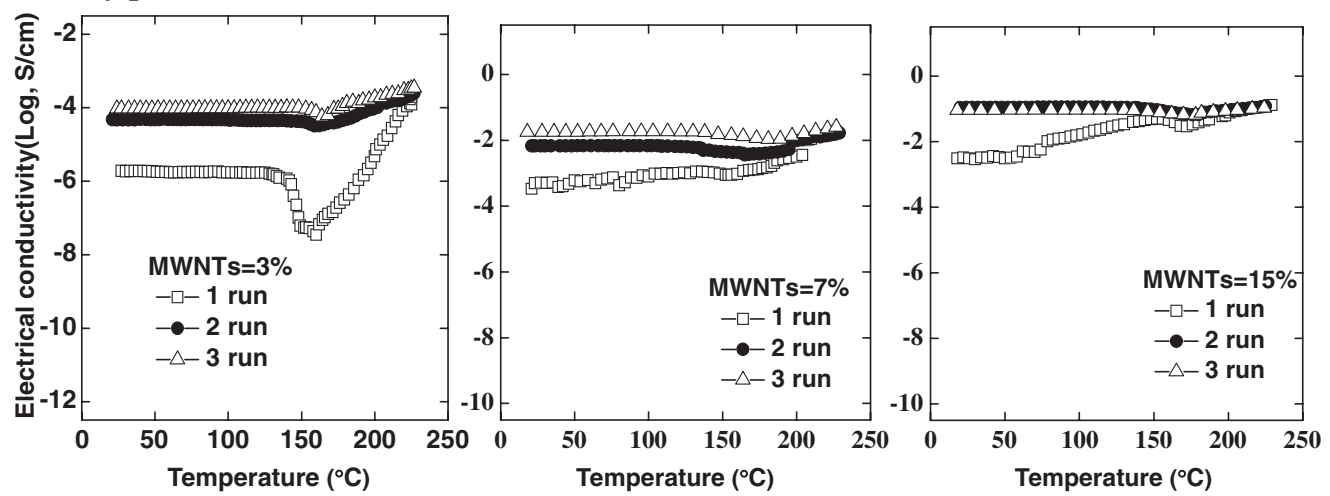

Figure 7. Temperature dependence of electrical conductivity measured for UHMWPE-MWNT composites prepared by (a) using decalin (b) using paraffin with the MWNTs contents $3 \mathrm{wt} \%, 7 \mathrm{wt} \%$ and $15 \mathrm{wt} \%$.

the assumption that the densities of UHMWPE and MWNT are 0.96 and $2.0 \mathrm{~g} / \mathrm{cm}^{3}$, respectively. This supports the concept that under the gelation/crystallization process, most of the UHMWPE chains were crystallized on the surface of the MWNTs.

To obtain more conclusive evidence, further measurements of the electrical conductivity of the two kinds of composite were carried out as a function of temperature. Figure 7 shows the results measured for the composites comprising 3, 7 and $15 \mathrm{wt} \%$ MWNT content. At temperatures less than he melting point of UHMWPE ( $\mathrm{ca} .150^{\circ} \mathrm{C}$ ), except in the case of the composites with $15 \%$ MWNT content, the conductivity of the original composites prepared using paraffin showed higher conductivity than that of the original composites prepared using decalin. However, the conductivity of the composite prepared in decalin increased drastically beyond $c a .150^{\circ} \mathrm{C}$, and attained a value similar to the value of paraffin at $c a .220^{\circ} \mathrm{C}$. Under the second and third heating runs, this small increase was confirmed for the composites prepared using both paraffin and decalin.

Detailed observations for both composites with $3 \mathrm{wt} \%$ MWNT content under the first heating run revealed that the conductivity decreased slightly at temperatures close to their melting point and attained their minimum value beyond $150^{\circ} \mathrm{C}$. This decrease in conductivity corresponds to an increase in resistivity, and this phenomenon is referred to as the socalled "positive temperature coefficient (PTC) effect", which is due to the thermal expansion of UHMWPE leading to the separation of adjacent fillers. Beyond $155^{\circ} \mathrm{C}$, the conductivity increased with further increasing temperature. This increase in conductivity corresponds to a decrease in resistivity, and this phenomenon is referred to as the "negative temperature coefficient (NTC) effect", which is due to migration of the fillers caused by active chain mobility under the melted flow of UHMWPE. However, the NTC effect for the composite prepared using paraffin was much smaller than the composite prepared with an admixture of carbon black. ${ }^{21}$ On the other hand, the electrical conductivity of the composite prepared using decalin increased drastically. Such drastic increase is attributed to the separation of UHMWPE from MWNT surfaces and to the formation of electrical networks from bare MWNTs. The temperature dependence of the conductivity under the second and third heating runs became less pronounced, since the networks formed from bare MWNTs were maintained under the cooling process, as shown in Figure 5b.

As for the composites prepared in decalin, the tem- 
(a) By decalin
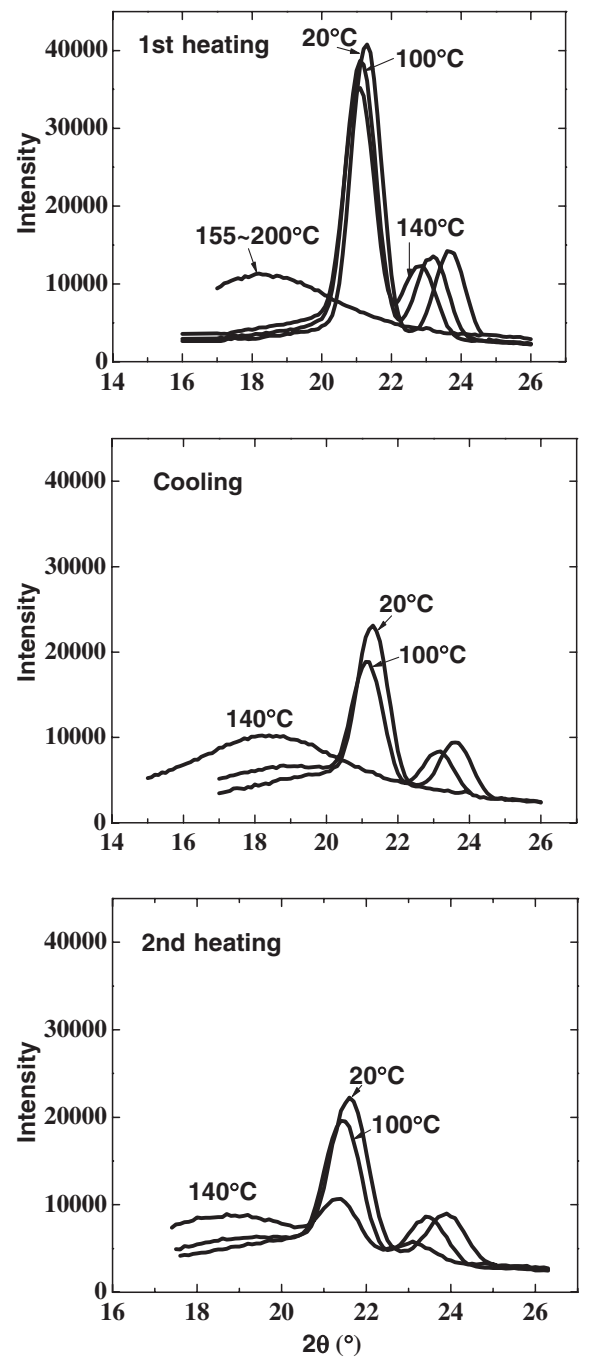

(b) By paraffin
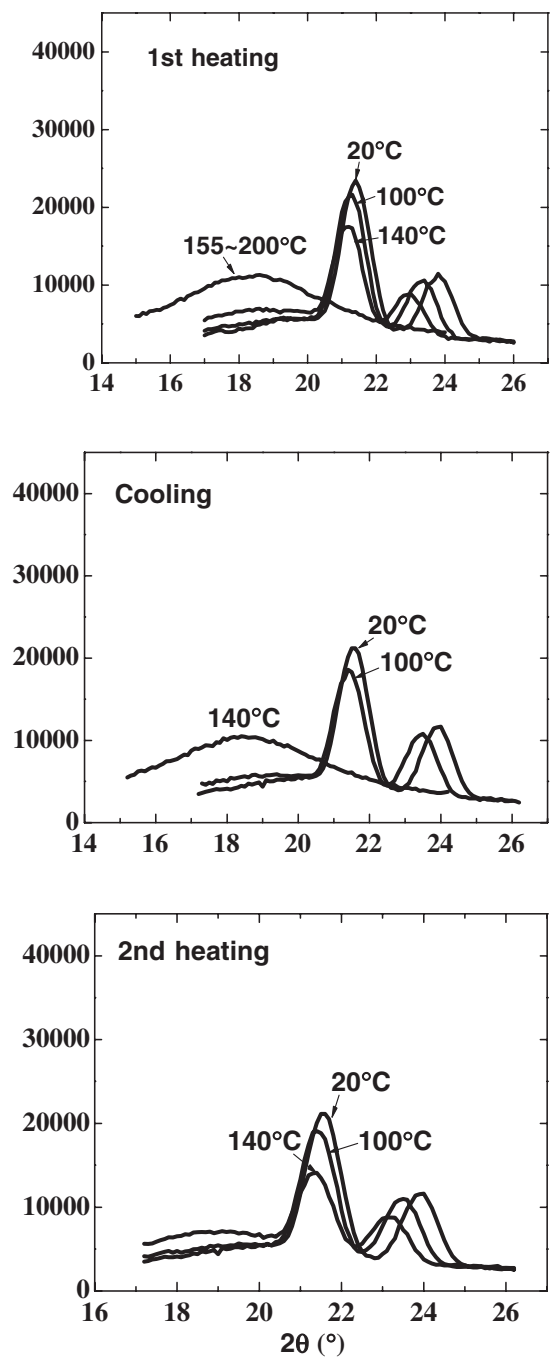

Figure 8. X-Ray diffraction intensity distribution curves at heating and cooling temperatures measured for of UHMWPE-MWNT composites with $7 \mathrm{wt} \%$ MWNT prepared by (a) using decalin (b) by using paraffin.

perature dependence of the electrical conductivity for the composite with $7 \mathrm{wt} \%$ MWNT loading is similar to that for the composite comprising $3 \mathrm{wt} \%$ MWNT content under the first run because of the low conductivity of the original composite. However, the temperature dependence was almost constant under the second and third runs, as discussed for the first run. The electrical conductivity of the original composite prepared with $15 \mathrm{wt} \%$ MWNT content in decalin, is higher than the conductivity values obtained for the composites with 3 and 7\% MWNT content at room temperature, and the increasing degree was much smaller than for the other composites. The increasing degree in the second and third runs was very small for the composite prepared in paraffin. This result supports the SEM images shown in Figure 5c and 5d, in which the morphology of the MWNTs dispersed in the composites before heat-treatment are almost the same as that after heat-treatment. Namely, the bare
MWNTs were dispersed within the UHMWPE matrix.

Figure 8 shows the temperature dependence of the $\mathrm{X}$-ray diffraction curves for the original composites containing $7 \mathrm{wt} \%$ MWNT content prepared in decalin (left side) and paraffin (right side). Under the heating process, the diffraction peak intensities decreased gradually up to $140^{\circ} \mathrm{C}$ and the peak top shifted to lower diffraction angles indicating thermal expansion along the $a$-axis. For temperatures $<140{ }^{\circ} \mathrm{C}$, the changes in the electrical conductivities of the compositions prepared in decalin and paraffin were within experimental error, as shown in Figure 7. However, when the X-ray diffraction peak from the UHMWPE crystallites disappeared on heating beyond $155^{\circ} \mathrm{C}$, the conductivity for the composite prepared in decalin increased drastically. Such a drastic increase is probably due to the formation of electrical networks of bare MWNTs following separation of the melted UHMWPE chains from the MWNT surfaces. These 
networks were maintained under the cooling process, and then the conductivity was almost constant under the second heating process. The same phenomenon was also confirmed for the composites prepared in paraffin, although a drastic increase was not observed because of the high conductivity at room temperature.

The question arises as to whether the drastic increase in conductivity beyond $200^{\circ} \mathrm{C}$ is attributed to the degradation reaction of UHMWPE and/or the progression of the reaction with the admixture of MWNTs. To check the stabilization effect of UHMWPE, TGA measurements were performed for the composites as a function of MWNT content under a nitrogen atmosphere. These TGA measurements minimized the mass loss brought about by MWNT oxidation, thereby allowing the UHMWPE to thermally decompose completely; the char yield was almost zero. The masses remaining at $520^{\circ} \mathrm{C}$ are almost entirely due to the remaining MWNTs (data not shown), and are consistent with the initial nanotube loading. A comparison of the TGA curves for composites comprising $1 \%$ MWNT and up to $15 \%$ MWNT loadings revealed that both TGA curves shifted to higher temperatures of about $8{ }^{\circ} \mathrm{C}$ and $12{ }^{\circ} \mathrm{C}$, respectively. This study reveals that there is a retardation of the onset of UHMWPE decomposition for the composites even with low MWNT loadings of about 1\%, and also there is an effect on the start and the end of the degradation, which is shifted to higher temperatures for the composite samples loaded with MWNT.

Figure 9 shows the temperature dependence of the storage $\left(E^{\prime}\right)$ and loss $\left(E^{\prime \prime}\right)$ moduli for the composites with MWNT content of $10 \mathrm{wt} \%$ prepared in decalin and paraffin. The measurements were performed for the original composites and heat-treated composites. Similar results were obtained for the UHMWPE films prepared in both solvents. In the given temperature range, $E^{\prime}$ of the original composite prepared in decalin is highest among the other three composites. $E^{\prime}$ of the composite prepared in decalin became lower by heattreatment, since the crystallinity changed from $\mathrm{ca} .88$ to $c a .62 \%$. The crystallinities were calculated on the basis of the peak area of the DSC curve by assuming the heat of fusion for $100 \%$ crystallinity to be 245.3 $\mathrm{J} / \mathrm{g},{ }^{22}$ as discussed later (see Figure 13). The values of $E^{\prime}$ (and also $E^{\prime \prime}$ ) were almost equal to the values of the composites prepared in paraffin, because of small changes in crystallinity from $c a$. 68 to $64 \%$. Incidentally, $E^{\prime}$ for the composites prepared in paraffin were hardly affected by the heat-treatment, since the original composite prepared in paraffin was molded at high temperature to prepare film sheets, where the crystallites of the composites before and after heattreatment were almost the same.

The temperature dependence of $E^{\prime \prime}$ for the original
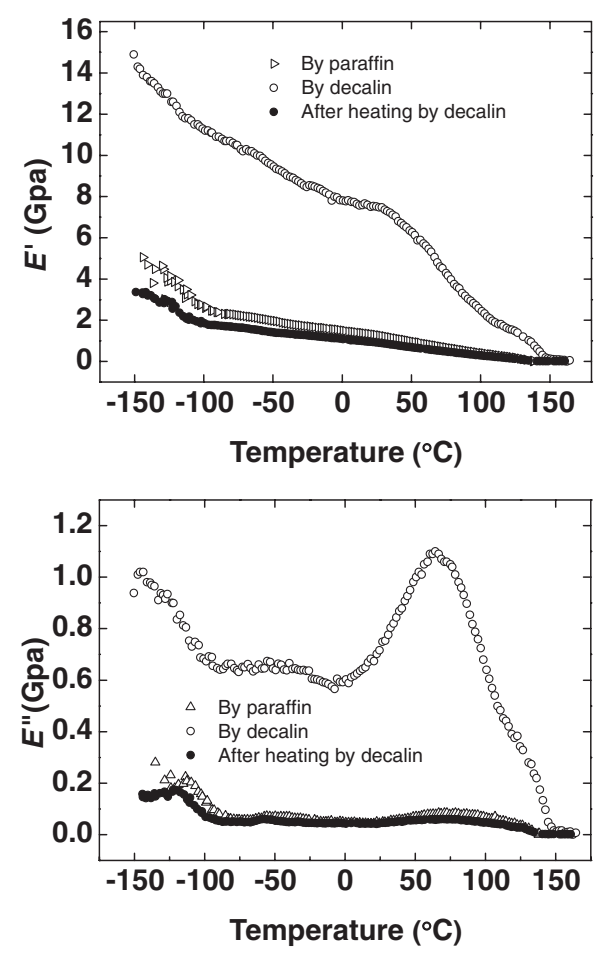

Figure 9. Temperature dependence of storage $\left(E^{\prime}\right)$ and loss $\left(E^{\prime \prime}\right)$ moduli for the composites prepared by decalin and paraffin solvents, in which MWNT content was $10 \mathrm{wt} \%$. (The measurements were done for original and heat-treeated composites.)

composite prepared in decalin showed a broad peak associated with the crystal dispersion termed as the $\alpha$ mechanism. However, this peak disappeared on heat-treatment, due to a decrease in the crystallinity. For the composites prepared in paraffin, no clear dispersion was observed because of very low crystallinity.

A big problem for the composites prepared in paraffin was that no elongation of the specimens was observed, while the composite prepared in decalin could be elongated up to 100 times. However, the conductivity was very low when the admixed MWNT content was less than $10 \mathrm{wt} \%$, and then changes in conductivity and mechanical properties were investigated by using composites elongated up to 50 times. The following discussion was conducted for the undrawn and drawn (50 times) composites prepared in decalin. Incidentally, the composites used for the test specimens in this discussion were prepared by gelation/crystallization at $60^{\circ} \mathrm{C}$ and the resultant gels were dried at the same temperature.

Figure 10 showed the electrical conductivity of the undrawn and drawn composites as a function of MWNT content measured at room temperature. The difference in conductivity between the undrawn and drawn composites was very small for the given MWNT contents. The electrical conductivity of the composites increased by 8 orders of magnitude from 


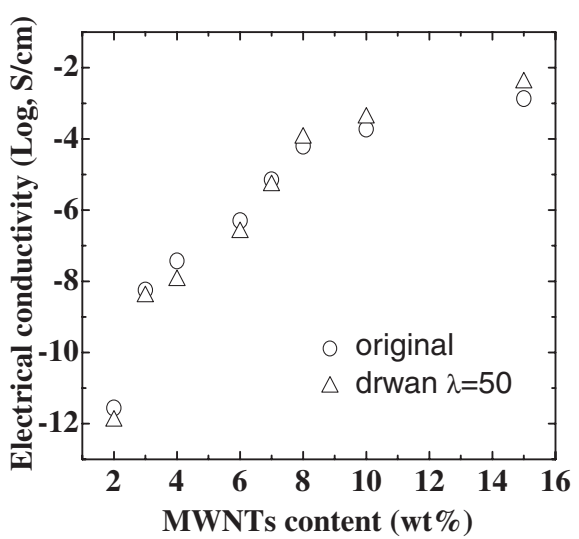

Figure 10. Electrical conductivity of composite gel films as a function of MWNT content ( $\lambda=1$ and 50).

$10^{-12} \mathrm{~S} / \mathrm{cm}$ to $10^{-4} \mathrm{~S} / \mathrm{cm}$ with increasing MWNT content up to $8 \mathrm{wt} \%$. The conductivity tended to increase gradually when the MWNT content increased from 8 to $15 \mathrm{wt} \%$. This indicated a typical percolation transition behavior. According to traditional percolation theory, ${ }^{23}$ the percolation threshold of the MWNT-UHMWPE composite was $3 \mathrm{wt} \%$ corresponding to a volume fraction of about $1.52 \%$, which was much lower than normal polyethylene composites with carbon black or carbon fibers. ${ }^{24}$ This result is attributed to the greater aspect ratio for MWNTs than for either carbon black or carbon fibers. As the mass fraction increased to the percolation threshold, the electrical conductivity increased sharply as conductive paths began to form. The electrical conductivity reached up to $10^{-2} \mathrm{~S} / \mathrm{cm}$ for composites with $15 \%$ MWNT content. However, further increases in the MWNT content did not improve the uniform dispersion and the aggregation of MWNTs hampered the increase in conductivity. Here, it may be noted that the electrical conductivities for the original undrawn composite were higher than those for the composites prepared by quenching and evaporating the solvent at room temperature, as shown in Figure 4. This was confirmed as a result of the thickness of the UHMWPE layers on the MWNT surfaces. The crystallization of UHMWPE on the MWNT surfaces became less pronounced as the temperature increased. Actually, the cooling of the original composite to room temperature occurred from the melt state, such that the crystallization of UHMWPE did not occur on the MWNT surfaces, as shown in Figure 5a and b.

As can be seen in Figure 10, the conductivity of the drawn composite $(\lambda=50)$ was slightly higher than that of the undrawn composite when the MWNT content was below 8\%. However, the difference in the electrical conductivity values between the undrawn and drawn composites for varying MWNT content is certainly very small. No decrease of conductiv-
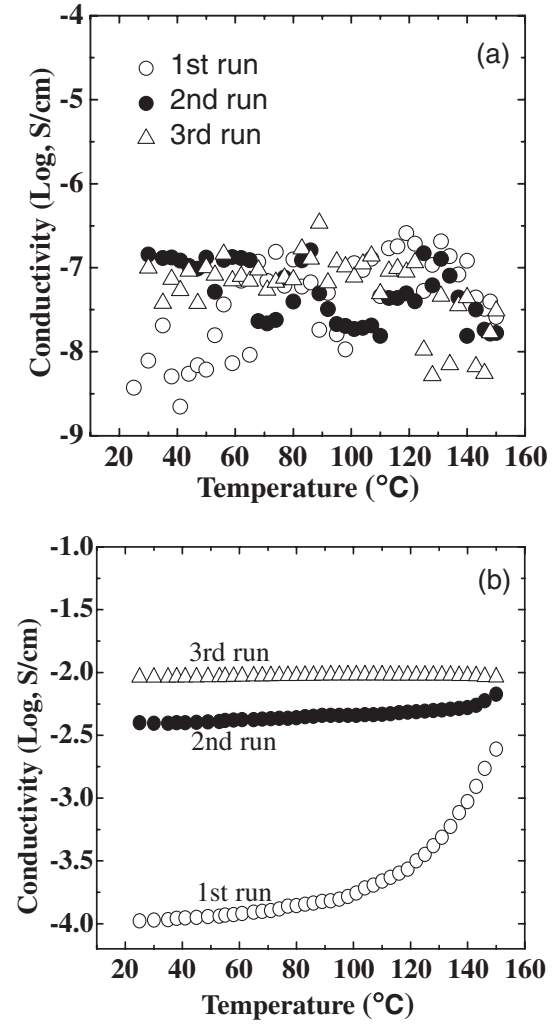

Figure 11. Temperature dependence of electrical conductivity for the elongated film at $\lambda=50$ with $3 \%$ (a) and $8 \%$ (b) MWNTs.

ity by elongation is one of characteristics of flexible MWNTs as fillers within the UHMWPE matrix. According to our previous report, ${ }^{15}$ the conductivity of the UHMWPE-MWNT composite with $15 \mathrm{wt} \%$ MWNT content was measured as a function of draw ratio up to 100 times. The experiments indicated that the networks of MWNTs were highly aligned with the oriented polyethylene fibrils along the elongation direction, and the orientation of MWNTs in the composite with an MWNT loading of more than $8 \%$ allowed a tight contact between MWNTs to promote a greater mobility of charge. Namely, the continuous networks of MWNTs formed most of the effective conductive paths in the drawn composite films. This characteristic was different from the composites filled with typical electrically conductive particles such as carbon black, as discussed previously.

Furthermore, the temperature dependence on the electrical conductivity for the elongated UHMWPEMWNT composite films with $3 \%$ and $8 \%$ MWNT content was investigated. The results for three heating cycles (1st, 2nd and 3rd run) in the temperature range of 25 to $150^{\circ} \mathrm{C}$ are shown in Figure 11. For the composite containing $3 \mathrm{wt} \%$ MWNT (corresponding to the percolation threshold), the electrical conductivity was irregularly scattered for the three runs in the given temperature range. Since the MWNT content was crit- 

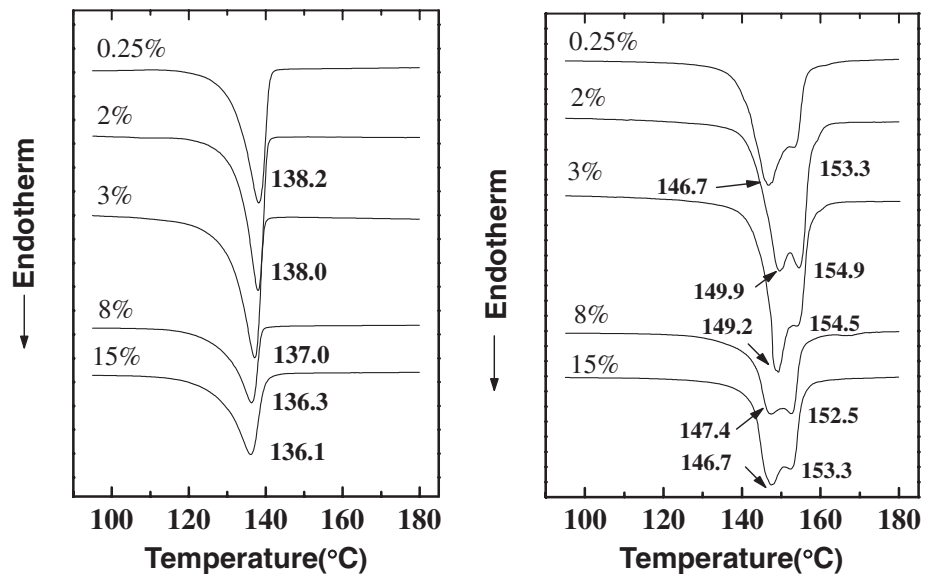

Figure 12. (a) DSC curves for the undrawn UHMWPE-MWNT composite; (b) for the elongated composite films at $\lambda=50$.

ical for the formation of a conductive network, it was thought that the hopping transport between the disconnected (or weakly connected) parts of the network induced by heat-treatment was unstable.

Comparing with the composite films with a $3 \mathrm{wt} \%$ MWNT loading, the stable conductivity of the composite film containing $8 \mathrm{wt} \% \mathrm{MWNT}$ in the given temperature range indicated the formation of a continuous network of MWNTs in the drawn composite. Obviously, a sharp increase in the conductivity occurred under the first run when the temperature was over $120^{\circ} \mathrm{C}$. In the second run, the conductivity tended to increase slightly. During the first run, the increase in conductivity beyond $100^{\circ} \mathrm{C}$ is due to the fact that part of the UHMWPE chains were maintained on the MWNT surface under elongation of the original composites at $135^{\circ} \mathrm{C}$, and the residual UHMWPE was separated from the MWNT surface under the first heating run.

Figure 12a and $b$ show DSC curves for the undrawn and drawn composites, respectively, with the indicated MWNT contents. It is seen that the endothermic peak associated with the melting point slightly shifted to a lower temperature and the endotherm peak became broader as the MWNT content increased. This suggested that the MWNTs hampered the crystallization of UHMWPE. The crystallinity of the composite film with different MWNTs will be discussed later.

Comparing the DSC profiles for the undrawn UHMWPE-MWNT composite films as shown in column (a), the endotherm peaks of the UHMWPE crystallites shown in column (b) became sharper, and the melting points shifted about $10^{\circ} \mathrm{C}$ to a higher temperature, was attributed to the orientated crystallization of the UHMWPE crystallites. The melting points measured for the drawn films were higher than the equilibrium melting point $\left(145.5^{\circ} \mathrm{C}\right)$ reported by Flory and Vrij. ${ }^{25}$ This abnormally high melting point

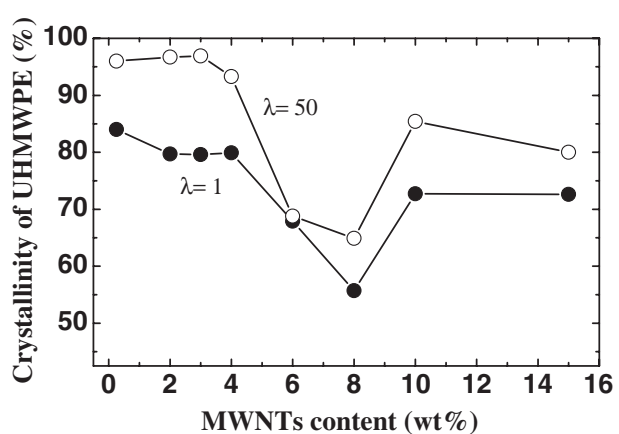

Figure 13. Crystallinity degree of the composite films at $\lambda=1$ and 50 calculated from DSC measurements.

was thought to be due to the polymer chains in the melting state that retained the extended-chain arrangement. This entropy in fusion was obviously smaller than the value calculated for the random coils in the melting state.

The corresponding crystallinity calculated from the DSC measurements for the undrawn and drawn films is shown in Figure 13. The values were calculated from the ratio of the measured $\Delta h$ to $\Delta h_{\mathrm{f}}$ values of $100 \%$ crystalline polyethylene $(245.3 \mathrm{~J} / \mathrm{g}) .^{22}$ The crystallinity of the undrawn composite film decreased slightly when the MWNT content was below $4 \%$. The crystallinity decreased sharply due to the MWNTs hampering the crystallization of UHMWPE. It seemed there existed a critical content influencing the crystallization. Similar to the percolation threshold of conductivity, the crystallinity decreased slowly below the critical content, while above the critical content, the formation of the continuous networks of MWNTs disturbed the crystallization of the polymer causing the crystallinity to decrease quickly. This tendency is probably attributed to the different thermal diffusions between the composites containing MWNTs above and below critical contents, since the thermal diffusion of the composites above the critical content 

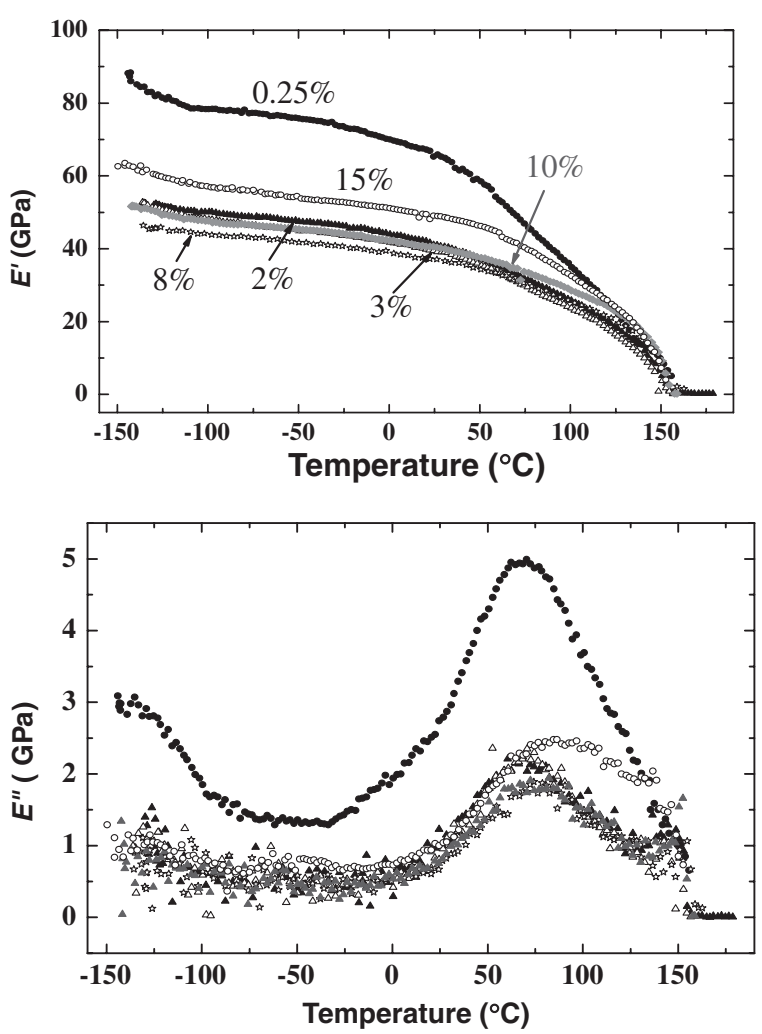

Figure 14. Temperature dependence of storage $\left(E^{\prime}\right)$ and loss $\left(E^{\prime \prime}\right)$ moduli of elongated films $(\lambda=50)$.

is higher. However, the detailed quantitative analysis must be taken into consideration. Furthermore, it was noticed that the crystallinity began to increase when the MWNT loading was more than $8 \%$. It is suggested that the addition of excessive MWNTs just led to aggregation of the MWNT networks. The aggregated MWNTs easily separated from the polymer matrix, causing the crystal domains and crystallinity to increase.

Figure 14 shows the temperature dependence on the storage $\left(E^{\prime}\right)$ and loss $\left(E^{\prime \prime}\right)$ moduli for the composite films at the drawn ratio of 50 -fold. The $E^{\prime}$ modulus decreased with increasing MWNT content, while the MWNT content lower than $8 \mathrm{wt} \%$, a reversal in trend occurred for the composites containing more than $8 \mathrm{wt} \%$ MWNTs. As shown in Figure 13, the crystallinity of the composite film with $8 \%$ MWNT loading was the lowest, which could lead to the smallest $E^{\prime}$. For the composite with $15 \mathrm{wt} \%$ MWNT content, the conductivity reached up to $10^{-1} \mathrm{~S} / \mathrm{cm}$, and the modulus was higher than $50 \mathrm{GPa}$ at $c a .25^{\circ} \mathrm{C}$, indicating that a polymeric material with very high modulus and relative high conductivity was successfully prepared.

Due to the high crystallinity of the UHMWPE composite film, its temperature dependence on $E^{\prime \prime}$ revealed that the magnitude of the $\beta$ relaxation peak (around $-50^{\circ} \mathrm{C}$ ) associated with the amorphous dis- persion $^{26,27}$ was very small. The position of the $\alpha$ relaxation peak associated with the crystal disper$\operatorname{sion}^{26-29}$ of UHMWPE (around $50-100^{\circ} \mathrm{C}$ ), slightly shifted to a lower temperature with increasing MWNT content, indicating the disorder of the UHMWPE crystallites.

\section{CONCLUSION}

UHMWPE and MWNT composites were prepared in decalin and paraffin solvent. Various analytical measurements were performed for the original and heat-treated specimens. Sample preparation by decalin was adopted as an example of gelation/crystallization from solution, while the sample preparation by paraffin was adopted instead of films molded at the melt state, since the film preparation by melting of UHMWPE powder at high temperature was impossible because of extremely high viscosity of the melt state. The drastic increase in electrical conductivity occurred at low MWNT content for the composite prepared in paraffin. On the other hand, the electrical conductivity of the composite prepared in decalin, after evaporation of the solvent at room temperature, increased slightly up to $8-10 \%$ MWNT content, and the drastic increase could be achieved by the heat-treatment, where the obtained values are close to those of the composite prepared in paraffin. Such different behavior in the conductivity with respect to MWNT content in the composites was attributed to the quite different morphology of the original components prepared in decalin and paraffin. SEM observations revealed that the MWNTs within the matrix of the composite prepared in decalin were covered by UHMWPE, and their average diameters became much thicker than the original diameters of the MWNTs, indicating that most of the UHMWPE chains were crystallized on the MWNT surface acted as nuclear growth of UHMWPE under gelation process. However, the composite was heated beyond the melting point of UHMWPE, the melted UHMWPE chains did not crystallized on the MWNT surface. SEM observation revealed the dispersion of bare MWNTs in the UHMWPE matrix. Therefore the conductivity at the same MWNT content increased drastically after the heat treatment. On the other hand, an image of the cross section of the composite prepared in paraffin solvent is similar to the diameters of MWNTs in the matrix maintained almost the same diameter of the original MWNTs, indicating that most of the UHMWPE chains were not crystallized on the surface of the MWNTs, and these MWNTs were dispersed uniformly in the matrix. The morphology was similar to that of the heat-treated composite prepared by decalin. Hence, the conductivity of the original compo- 
sites at the same MWNT content was higher than that of the original composite prepared by decalin, which was almost equal to the conductivity of the heat treated composite.

The composite prepared in decalin was elongated up to 50-fold, while the composite prepared in paraffin was not elongated. For the electrical conductivity of the composites, which were prepared in decalin and the solvent was evaporated at $60^{\circ} \mathrm{C}$, the percolation threshold was found for composites with $3 \mathrm{wt} \%$ MWNT content. For the composite with 3 wt $\%$ MWNT, however, the electrical conductivity was irregular scattered in the temperature range of 25 to $150^{\circ} \mathrm{C}$ during heating cycles. On the other hand, the conductivity of the composite film containing more than $8 \mathrm{wt} \%$ MWNT smoothly increased in the given temperature range, due to the stable structure of the composites and the formation of continuous networks of MWNTs in the composites. The UHMWPE crystallinity of the drawn composite with $8 \mathrm{wt} \%$ MWNT loading is the lowest and provided the lowest Young's modulus.

For the drawn UHMWPE-MWNT composite film with $15 \mathrm{wt} \%$ MWNT content, the conductivity reached up to $10^{-1} \mathrm{~S} / \mathrm{cm}$ and the modulus was higher than $50 \mathrm{GPa}$ at $\mathrm{ca} .25^{\circ} \mathrm{C}$, indicating that a polymeric material with very high modulus and relatively high conductivity was successfully prepared.

\section{REFERENCES}

1. J. P. Salvetat, J. M. Bonard, N. H. Thomson, A. J. Kulik, L. Forro, W. Benoit, and L. Zuppiroli, Appl. Phys. A: Mater. Sci. Process., 69, 255 (1999).

2. M. M. J. Treacy, T. W. Ebbesen, and T. M. Gibson, Nature, 381, 680 (1996).

3. E. W. Wong, P. E. Sheenan, and C. M. Lieber, Science, 277, 1971 (1997).

4. M. Cadek, J. N. Coleman, V. Barron, K. Hedicke, and W. J. Blau, Appl. Phys. Lett., 81, 5123 (2002).

5. A. Koganemaru, Y. Bin, Y. Agari, and M. Matsuo, $A d v$. Funct. Mater., 14, 842 (2004).

6. M. J. Biercuk, M. Llaguno, M. Radosavljevic, J. K. Hyun, A. T. Johnson, and J. E. Fischer, Appl. Phys. Lett., 80,
2767 (2002).

7. K. P. Ryan, M. Cadek, V. Nicolosi, S. Walker, M. Ruether, A. Fonseca, J. B. Nagy, W. J. Blau, and J. N. Coleman, Synth. Met., 156, 332 (2006).

8. P. Potschke, S. M. Dudkin, and I. Alig, Polymer, 44, 5023 (2003).

9. Q. Zhang, D. R. Lippits, and S. Rastogi, Macromolecules, 39, 658 (2006).

10. B. E. Kilbride, J. N. Coleman, P. Fournet, M. Cadek, A. Drury, and W. J. Blau, J. Appl. Phys., 92, 4024 (2002).

11. J. P. Salvetat, A. D. Briggs, J. M. Bonard, R. R. Bacsa, A. J. Kulik, T. Stöckli, N. A. Burnham, and L. Forró, Phys. Rev. Lett., 82, 944 (1999).

12. S. L. Ruan, P. Gao, X. G. Yang, and T. X. Yu, Polymer, 44, 5643 (2003).

13. E. T. Thostenson and T. W. Chou, J. Phys. D: Appl. Phys., 35, L77 (2002).

14. Q. Chen, Y. Bin, and M. Matsuo, Macromolecules, 39, 6528 (2006).

15. Y. Bin, M. Kitanaka, D. Zhu, and M. Matsuo, Macromolecules, 36, 6213 (2003).

16. M. Matsuo, K. Inoue, and N. Abumiya, Sen-i-Gakkaishi (Fiber), 36, 696 (1984).

17. P. Smith and P. J. Lemstra, J. Mater. Sci., 15, 505 (1980).

18. P. Smith, P. J. Lemstra, J. P. L. Pippers, and A. M. Kiel, Colloid. Polym. Sci., 258, 1070 (1981).

19. T. Kanamoto, A. Tsuruta, K. Tanaka, M. Takeda, and R. S. Porter, Polym. J., 15, 327 (1983).

20. T. Ogita, K. Kawahara, K. Soga, and M. Matsuo, Polymer, 33, 689 (1992).

21. Y. Bin, C. Xu, Y. Agari, and M. Matsuo, Colloid Polym. Sci., 277, 452 (1999).

22. J. Brandrup, E. H. Immergut, and E. A. Grulke, "Polymer handbook," 4th ed., Wiley, New York, 1999.

23. D. Stauffer and A. Aharony, "Introduction to Percolation Theory," Taylor and Francis, London, 1994.

24. M. Weber and M. R. Kamal, Polym. Compos., 18, 711 (1997).

25. P. J. Flory and A. Vrij, J. Am. Chem. Soc., 85, 3548 (1963).

26. H. Kawai, T. Hashimoto, S. Suehiro, and K. Fujita, Polym. Eng. Sci., 24, 361 (1984).

27. M. Matsuo, Y. Bin, C. Xu, L. Ma, T. Nakaoki, and T. Suzuki, Polymer, 44, 4325 (2003).

28. M. Matsuo, C. Sawatari, and T. Ohhata, Macromolecules, 21, 1317 (1988).

29. M. Matsuo, L. Ma, M. Azuma, C. He, and T. Suzuki, Macromolecules, 35, 3059 (2002). 